\title{
ROLE OF FIBEROPTIC BRONCHOSCOPY IN DIAGNOSIS OF SMEAR-NEGATIVE PTB
}

\author{
Chukka Bharat ${ }^{1}$, Kondala Rao Kola², Ram Prasad Reddy Nandyala ${ }^{3}$
}

${ }_{1}^{1}$ Senior Resident, Department of Pulmonary Medicine, Andhra Medical College, Visakhapatnam, Andhra Pradesh, India. 2 Associate Professor, Department of Pulmonary Medicine, GSL Medical College, Rajahmundry, Andhra Pradesh, India. 3Junior Resident, Department of Pulmonary Medicine, GSL Medical College, Rajahmundry, Andhra Pradesh, India.

\section{ABSTRACT}

\section{BACKGROUND}

The most common method for diagnosing TB worldwide remains sputum smear microscopy. Sputum smears do not reveal acidfast bacilli (AFB) in all patients. Fiberoptic bronchoscopy (FOB) can provide alternative respiratory specimens for diagnosis.

\section{MATERIALS AND METHODS}

A hospital-based cross-sectional observational study was conducted. Patients having cough with expectoration for more than two weeks with clinical and radiological evidence of PTB along with sputum smear for AFB, tested negative between October 2014 and July 2016 were taken for study. FOB will be performed in all those individuals and the collected specimens will be examined for AFB under the microscope. Statistical analysis was performed by using Microsoft Excel-2007 and IBM SPSS software version 20.0.

\section{RESULTS}

Bronchoscopy was performed on 51 cases of clinical and radiological suspects of PTB. Most of the study participants were male s (67\%). Mean age group was $47.3 \pm 18.01$. In between 21 and 60 years age, 38 patients were present. Cough was the most common presenting symptom with $93.5 \%$ of the patients followed by breathlessness, fever, chest pain and haemoptysis. 17 patients had positive post bronchoscopy AFB among 51; 3 patients were diagnosed as PTB based on their clinical and radiological features. Bronchoscopy-guided biopsy confirmed 4 malignancies and 1 benign polyp. Bronchial washings cytology diagnosed 1 adenocarcinoma. CT-guided FNAC/ biopsy confirmed diagnosis in 6 patients, 5 malignancies and 1 lung abscess.

\section{CONCLUSION}

Bronchoscopy is a useful tool in early diagnosis of smear negative PTB. It will help in preventing under-diagnosis as well as overdiagnosis of the disease.

\section{KEY WORDS}

Bronchoscopy, TB, Smear Negative.

HOW TO CITE THIS ARTICLE: Bharat C, Kola KR, Nandyala RPR. Role of fiberoptic bronchoscopy in diagnosis of smear-negative PTB. J. Evolution Med. Dent. Sci. 2018;7(28):3178-3182, DOI: 10.14260/jemds/2018/715

\section{BACKGROUND}

The most common method for diagnosing TB worldwide remains sputum smear microscopy. Sputum smears do not reveal acid-fast bacilli (AFB) in all patients. Mycobacterial cultures take at least six to eight weeks' time for confirming the diagnosis and thereby a valuable time is lost. Fiberoptic bronchoscopy (FOB) can provide alternative respiratory specimens for diagnosis, especially from specific sites that are suspected by radiological testing for involvement of PTB when expectoration has repeatedly failed, because sputum is absent. FOB diagnostic yield is more useful in the diagnosis of endobronchial TB. Results of bronchial washing specimens with AFB smears and tissue specimens from bronchoscopic biopsies are usually received within one week, thus enabling rapid diagnosis before the availability of confirmation from sputum cultures of sputum smear-negative PTB patients.

'Financial or Other Competing Interest': None.

Submission 26-05-2018, Peer Review 24-06-2018,

Acceptance 30-06-2018, Published 09-07-2018.

Corresponding Author:

Dr. Kondala Rao Kola,

Department of Pulmonary Medicine,

GSL Medical College,

Rajahmundry-533296,

Andhra Pradesh, India.

E-mail: kkraodr@gmail.com

DOI: $10.14260 /$ jemds $/ 2018 / 715$
Aims and Objectives of the Study

1. To analyse the role and effects of Fiberoptic Bronchoscopy (FOB) in early diagnosis of PTB in smear negative cases.

2. To study the diagnostic efficacy of microscopic examination of bronchial aspirate obtained by fiberoptic bronchoscope in patients with smear negative PTB.

3. To compare the diagnostic yield of sputum sample versus bronchial aspirate in smear-negative PTB.

\section{MATERIALS AND METHODS}

A hospital-based cross-sectional observational study was conducted in the Department of Respiratory Medicine. After getting ethical clearance, all patients who are above 14 years of age having cough along with expectoration for more than two weeks with clinical and radiological evidence of PTB along with sputum smear for AFB tested negative between October 2014 and July 2016 were taken for study. Patients on ATT are excluded from the study.

After detailed clinical history thorough physical examination and routine investigations, sputum samples will be sent for AFB staining. Patients with positive sputum reports will be started with ATT as per the RNTCP guidelines. Smear negative patients with clinical and radiological features in favour of PTB will be assessed for the fitness to undergo fiberoptic bronchoscopy. FOB will be performed in all those individuals and bronchial aspirate, bronchoalveolar lavage and post bronchoscopy sputum will be collected from 
the patient. The collected specimens will be examined for AFB under the microscope.

The results of all the reports will be compiled and statistically analysed using Microsoft Excel-2007 and IBM SPSS software version 20.0. Descriptive statistical data was presented in the form of mean \pm standard deviation and percentages. Data was tabulated and graphically represented. Diagnostic test was performed to assess the sensitivity and specificity.

\section{RESULTS}

The age group among the patients studied varied from 16 to 81 with mean age of 47.31 with a standard deviation of 18.01 and most of them falling into the age group of 41 to 60 years. Of the 51 patients, 34 (66.66\%) were males and 17 (33.33\%) were females.

Cough is the most common presenting symptom in $96.08 \%$ of total study group, next are breathlessness (84.31\%), fever (64.74\%), chest pain (27.45\%), haemoptysis (21.57\%), Graph-1.

Of the total 51 cases of the study group, 16 had past history of PTB and took ATT. Among 20 patients with PTB, 6 cases had relapse. 5 patients had healed TB, diagnosed as old TB (sequelae). Of the 26 patients in non-TB group, 5 had earlier TB. Total 6 patients had history of contact with established case of PTB. Of them 4 patients came out as PTB. Even though 16 patients having no history of contact, developed TB.

Among study population of 51 patients, 16 patients (31.37\%) had cavitary lesions. 14 (27.45\%) had consolidation, 2 (3.92\%) had reticulonodular picture, 1 (1.96\%) had pleomorphic pattern, 4 (7.84\%) had mass lesions, 4 (7.84\%) had fibrosis, 2 (3.92\%) had reticular pattern, 5 (9.80\%) had bronchiectatic changes and 3 (5.88\%) had collapse, Graph- 2, 3.

Post bronchoscopy aspirate came out as AFB positive in $17(33.33 \%)$ of the total 51 patients. In remaining 34 (66.66\%) post bronchoscopy aspirates for AFB came negative. Among total 20 diagnosed PTB cases, 17 were diagnosed by post bronchoscopy sample for AFB. Post bronchoscopy AFB helped in diagnosis of $85 \%$ of total PTB cases, remaining $15 \%$ were diagnosed based on clinical/radiological basis, Table-1.

Bronchoscopy-guided washings, biopsy gave histological diagnosis in 23 out of the 51 patients of the study group. Out of them, 17 were AFB positive in bronchial washings. On bronchoscopy-guided biopsy histological examination revealed 4 malignant lesions and 1 benign (polyp) and 1 bronchial washings cytology showed features of papillary adenocarcinoma. CT-guided FNAC/ biopsy gave conclusive diagnosis in 5 malignant pathology and 1 inflammatory pathology (lung abscess). One case is diagnosed as topical pulmonary eosinophilia based on history of presentation, radiological findings, elevated total serum IgE and absolute eosinophil counts. Other diagnosis was based on clinical, radiological and absence of AFB in bronchial washings, Graph-4.

PTB was the most common diagnosis observed in 20 out of 51 patients, 5 had old TB sequelae, 8 had bronchiectasis, 9 had malignancy, 2 had lung abscess, 2 had non-resolving pneumonia, 1 had tropical pulmonary eosinophilia, 1 had pulmonary alveolar haemorrhage, 1 had fungal pneumonia, 1 had DPLD and 1 had benign polyp, Graph-5.
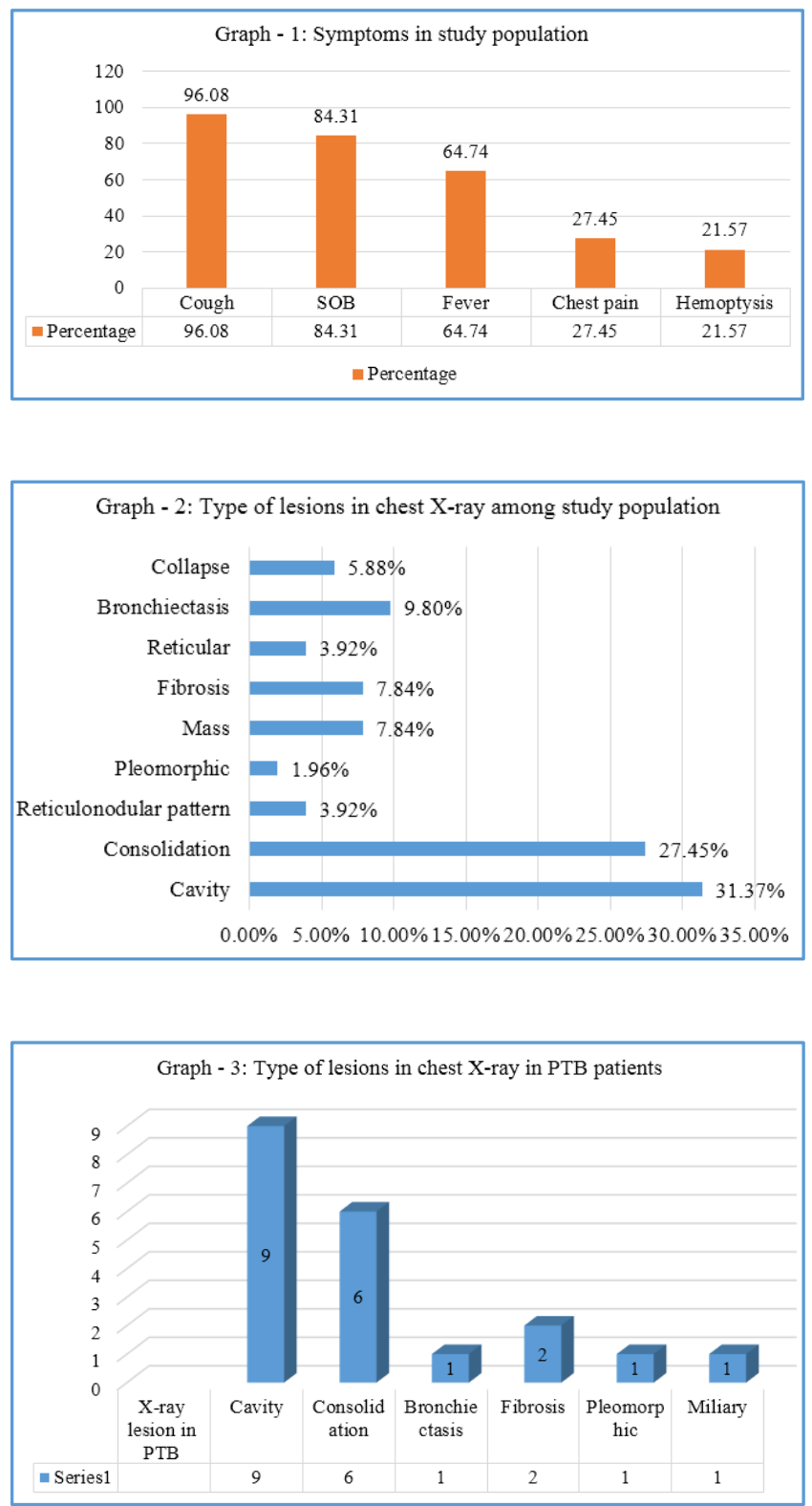

\begin{tabular}{|c|c|c|c|c|c|c|c|c|c|}
\hline & & $\begin{array}{c}\text { PTB } \\
\text { Present }\end{array}$ & $\begin{array}{c}\text { PTB } \\
\text { Absent }\end{array}$ & $\begin{array}{c}\text { True } \\
\text { Positives }\end{array}$ & $\begin{array}{c}\text { False } \\
\text { Negative }\end{array}$ & $\begin{array}{c}\text { True } \\
\text { Negative }\end{array}$ & $\begin{array}{c}\text { False } \\
\text { Positive }\end{array}$ & Sensitivity & Specificity \\
\hline $\begin{array}{c}\text { Bronchial } \\
\text { Aspirate }\end{array}$ & $\begin{array}{c}\text { AFB } \\
\text { Positive }\end{array}$ & 17 & 0 & \multirow{2}{*}{17} & \multirow{2}{*}{3} & \multirow{2}{*}{31} & \multirow{2}{*}{0} & \multirow{2}{*}{$85 \%$} & \multirow{2}{*}{$100 \%$} \\
\hline $\begin{array}{c}\text { Bronchial } \\
\text { Aspirate }\end{array}$ & $\begin{array}{c}\text { AFB } \\
\text { Negative }\end{array}$ & 3 & 31 & & & & & & \\
\hline
\end{tabular}



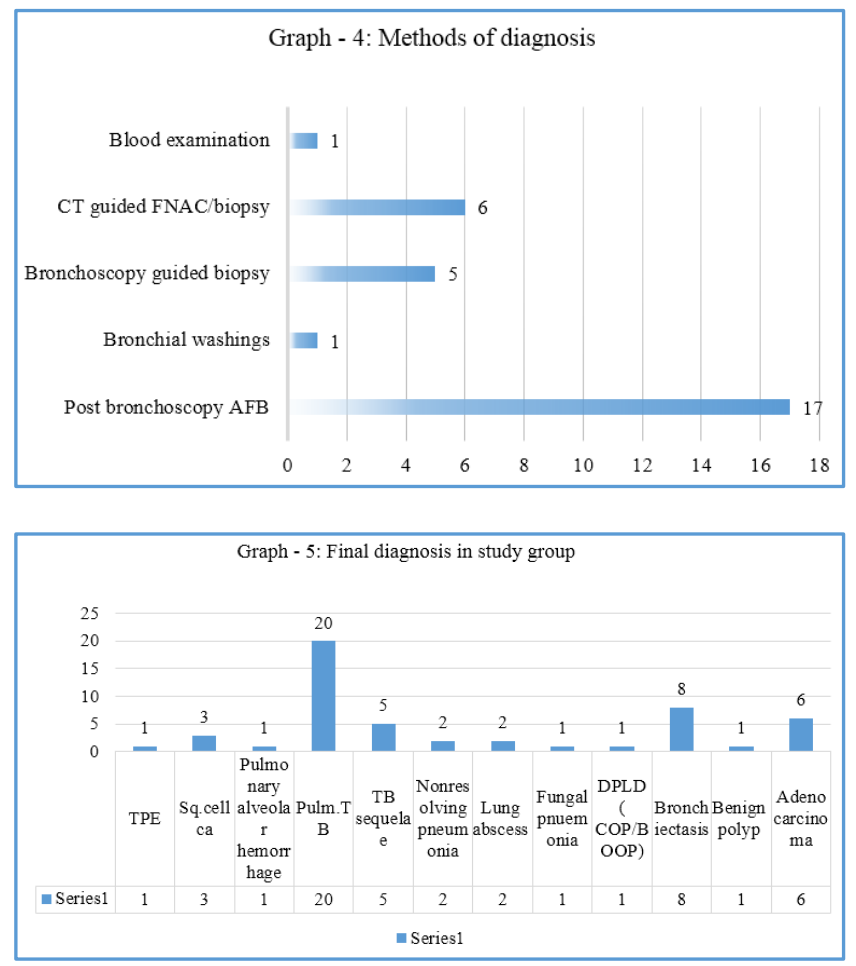

\begin{tabular}{|c|c|c|c|c|}
\hline $\begin{array}{c}\text { Presenting } \\
\text { Symptom }\end{array}$ & $\begin{array}{c}\text { Present } \\
\text { Study }\end{array}$ & $\begin{array}{c}\text { Shin et } \\
\text { al }\end{array}$ & $\begin{array}{c}\text { Khara et } \\
\text { al }\end{array}$ & $\begin{array}{c}\text { Bachh et } \\
\text { al }\end{array}$ \\
\hline Cough & $96.08 \%$ & $55.6 \%$ & $91.7 \%$ & $90.6 \%$ \\
\hline Fever & $64.74 \%$ & $21.4 \%$ & $61.9 \%$ & $68.0 \%$ \\
\hline Chest pain & $27.45 \%$ & $17.5 \%$ & $38.8 \%$ & $14.6 \%$ \\
\hline Haemoptysis & $21.57 \%$ & $24.61 \%$ & $20.4 \%$ & $16.0 \%$ \\
\hline \multicolumn{5}{|c|}{ Table 2 } \\
\hline
\end{tabular}

\section{DISCUSSION}

TB is a major global health problem, ranging as curable killer number one in India today. More than one-third of 9 million people infected with TB each year are not diagnosed, not notified or not put under treatment. ${ }^{1}$ Too many people have undetected TB (TB) for too long, like an ice berg. Late detection of $\mathrm{TB}$ increases their risk of transmitting the disease to others. India's Revised National TB Programme has developed objectives for 2020 with milestones for each year, which are aligned with the end TB strategy. Among the priorities are the provision of Xpert MTB/RIF and other rapid and better diagnostics, free treatment for all forms of TB, both in the public sector and through special initiatives with private organisation, accelerated expansion of treatment for MDR-TB and expanding linkages with social welfare schemes including nutritional support. ${ }^{2}$

The initial diagnostic approach to PTB suspects is to demonstrate MTB in stained smears of expectorated sputum. In most of the $\mathrm{TB}$ centres, the bacteriological positive yield from sputum is $16 \%$ to $50 \%$ and large portion remain negative in spite of clinical profile, though radiological lesions are consistent with PTB. ${ }^{3}$ Early diagnosis of PTB prevents progression of disease, decreases morbidity and prevents spread of disease and permanent damage. Culture of sputum for acid fast bacilli (AFB) takes long time and a reliable serological test is not yet available. In such a situation, bronchoscopy has been studied for rapid diagnosis of TB in smear negative cases. In patients where AFB demonstration in expectorated sputum has repeatedly failed, bronchoscopy may be useful. Many studies showed that bronchoscopy could lead to immediate, accurate diagnosis in suspected smear negative cases. Bronchial aspirate and bronchoalveolar lavage along with tissue biopsy will improve to the diagnostic yield.

The present study is a cross-sectional observational study which consists of 51 patients, 17 (33.33\%) females and 34 $(62.96 \%)$ males who were clinical and/ or radiological suspects of PTB. Sputum for AFB was found to be negative by fluorescent microscopy in RNTCP affiliated designated microscopy centre. The male: female ratio in this study was $2: 1$. In many of the earlier studies, there is more preponderance of males in comparison to females. Balakrishna et al studied 70 cases, where $66 \%$ were males. ${ }^{4}$ In the study of Quaiser et al, $82.5 \%$ were males. ${ }^{5}$ The fewer percentage of females in different Indian studies may be due to sedentary lifestyles of females in India. As TB is still a social stigma in our country, fewer numbers of women visit the health facilities. That contributes to fewer percentage of females among the study participants.

Mean age of study group was 47.31 with standard deviation of \pm 18.01 . The mean age of males in study group was $48.91 \pm 13.16$, and that in females was $44.12 \pm 18.01$. In the study of Shin et al, the mean age of TB patients among the participants was $45.3 \pm 18.8 .^{6}$ In similar study of Quaiser, the mean age was $38.5 \pm 9.5$ years. 5 These observations of various studies and the present study suggests that TB affects mainly the young age group, who have highest work efficiency. That is the reason why case finding of TB is essential in a community. In symptomatic suspected cases of TB if sputum smear is not diagnostic, then these individuals may suffer and also spread the disease in the community. According to Alladi Mohan and SK Sharma, in areas with low transmission of TB $17 \%$ of the disease transmission could be attributed to smear negative and culture positive index patients. In this present study reports have been made to find a better tool, to identify the patients of TB in the pool of smear negative suspects. FOB, though an invasive procedure it is a very safe procedure and can be performed in this adult age group.

Detailed clinical history was taken in all study participants during the study. Cough was the most common presenting symptom, present in $96 \%$ of patients followed by shortness of breath in $84 \%$ of patients. Fever was not a very common presentation, observed in $64.7 \%$ of patients. Only $21 \%$ of cases presented with coughing of blood (Graph-1). In the study of Khara et al also cough was the predominant symptom, i.e. $91.7 \%$ among 289 study participants, fever in $61.9 \%$ and haemoptysis in $20.4 \%$ of cases. ${ }^{7}$ In the study of Bachh et al also, cough was the commonest presenting symptom accounting for $90.6 \%$ and haemoptysis was as low as $16 \% .{ }^{8}$ From this observation of the study, it is derived that clinical diagnosis in maximum cases of TB suspects was based on their presenting symptoms, mostly cough for more than two weeks, Table- 2 .

According to Alladi Mohan and SK Sharma, sputum smear negativity in TB cases are due to inadequate sputum sample at the time of examination. ${ }^{9}$ Out of the 51 patients in this study, 17 members were found to be positive for AFB in their FOB derived specimen. Among those individuals, $100 \%$ have cough. Study participants in the present study were distributed into two groups basing on their post bronchoscopy findings, viz. tubercular and non-tubercular. 
Symptoms like cough and fever were more in tubercular group and SOB, haemoptysis were more in non-tubercular group. This observation was found to be statistically significant.

Contact history of patients with established case of PTB were identified. Of the 51 patients analysed, 6 patients $(11.76 \%)$ had positive history of contact with established case of PTB. Among PTB group of 20 patients, 4 patients (20\%) had positive contact history in comparison to nontubercular group of 31 who had only 2 patients (6.4\%) with contact history. Study shows higher percentage of contact history in PTB group in comparison to non-tubercular group. Even though 16 patients of PTB group did not reveal any contact history, they developed TB. History of TB in study group, a total of 16 patients (31.37\%) had old history of PTB and ATT intake. Among 20 patients of TB group, 6 patients (30\%) had relapse. Excluding old TB patients, other diseases group had 5 relapse cases (19.23\%) among 26 patients. According to central TB division, the percentage of smear positive retreatment cases out of all smear positive cases is $24 \%$. This includes relapse, failure and treatment defaulter. Irregular intake of medication is another important risk factor. Thomas et al observed that $6 \%$ of their study population had initial isoniazid resistance and $26.6 \%$ of them had relapse after completing RNTCP category 1 treatment. 10 Global data shows that $32 \%$ of relapse cases are actually multi-drug resistant TB. However, percentage of relapse cases in current study group, i.e. $30 \%$ had close resemblance to that of RNTCP data and also on study done by Thomas et al mentioned above.

Chest radiographs were taken in all the study participants. Computerised tomography scan of chest was done in cases wherever feasible and necessary. Type, extent, zone-wise distribution of lesions, involvement of single or both the lungs on chest $\mathrm{x}$-ray were analysed. Also type of lesion and its relation to different diseases was analysed.

In study population of 51 patients, type of lesions on chest $\mathrm{x}$-ray were described. Of 51 patients, 16 patients (31.37\%) had cavitary lesions on their chest x-ray, 14 patients (27.45\%) had consolidation, 2 patients (3.92\%) had reticulonodular pattern, 1 patient $(1.96 \%)$ had pleomorphic pattern, 4 patients $(7.84 \%)$ had mass lesions, 4 patients (7.84\%) had fibrosis, 2 patients $(3.92 \%)$ had reticular pattern, 5 patients $(9.80 \%)$ had bronchiectatic changes and 3 patients (5.88\%) had collapse, Graph-2, 3. In similar study by Khara et al 5.5\% study subjects had cavitatory lesions, $27.3 \%$ had consolidation, $3.5 \%$ had infiltrative lesions, $10 \%$ had collapse, $3.5 \%$ had fibrosis, $23.5 \%$ had mass lesions, $2.4 \%$ had nodular lesions, $0.3 \%$ had reticular pattern and $3.5 \%$ had no abnormality detected. ${ }^{7}$

Among 51 patients of study group, 17 patients (33.33\%) had post bronchoscopy AFB positive who had previous sputum for AFB was negative by fluorescent microscopy, 34 (66.66\%) patients had post bronchoscopy sample of AFB negative. Among 20 diagnosed PTB cases, 17 were diagnosed through post bronchoscopy AFB. Post bronchoscopy AFB helped in diagnosis of $85 \%$ of total PTB cases, remaining $15 \%$ were diagnosed based on clinical and radiological findings. Sensitivity for bronchial aspirates for AFB is $85 \%$ and specificity is $100 \%$, Table- 1 . Bachh et al reported $35 \%$ post bronchoscopy AFB positivity in smear negative PTB suspects. ${ }^{8}$ In a study by Raj Kumar et al, bronchoscopy in immediate diagnosis of smear negative PTB reports 51.5\% post bronchoscopy AFB positivity. ${ }^{11}$ Study by Kalawat et al reported $64.70 \%$ post bronchoscopy AFB positivity, $82.35 \%$ post bronchoscopy culture for AFB positivity. ${ }^{12}$ In comparing with above studies, it is advisable to do bronchial washings culture for MTB along with post bronchoscopy AFB in order to get more diagnostic yield for TB.

On bronchoscopy-guided washings cytology and AFB examination, biopsy gave histological diagnosis in 23 cases (45.09\%) among total 51 patients. Out of 23, 17 were AFB positive on bronchial washings on bronchoscopy-guided biopsy. HPE revealed 4 malignant lesions and 1 benign polyp, and 1 bronchial washings cytology came out as papillary adenocarcinoma. CT-guided FNAC/ biopsy gave diagnostic yield in $11.76 \%$ of study participants, out of them 5 had malignant pathology, 1 had inflammatory pathology due to lung abscess, Graph-4. Bronchoscopy has been the most helpful investigation in this study group. $45.09 \%$ of patients in the study group were diagnosed with bronchoscopyguided procedures.

Most common diagnosis in the study group is PTB. 20 patients (39.21\%) were diagnosed as PTB, 5 (9.8\%) had old TB or TB sequelae, 8 (15.68\%) had bronchiectasis, 9 (17.64) had malignancy, 2 (3.92\%) had lung abscess, 2 (3.92\%) had non-resolving pneumonia, $1(1.96 \%)$ had tropical pulmonary eosinophilia, 1 (1.96\%) had pulmonary alveolar haemorrhage, 1 (1.96\%) had fungal pneumonia, 1 (1.96\%) had DPLD and 1 (1.96\%) had benign polyp. Pulmonary alveolar haemorrhage and DPLD was diagnosed based on HRCT and clinical findings. Among 4 cases of old PTB sequelae, 2 cases had fungal ball in the old tubercular cavity, Graph-5. Similar study by Kalawat et al, on 45 patients of sputum for AFB negative PTB suspects 34 patients were diagnosed with PTB. ${ }^{12}$ Among 34 PTB patients, 22 (64.7\%) patients were bronchial washings AFB positive, 28 (82.3\%) patients were positive for MTB as culture in bronchial washings. This study had 11 cases other than TB. They are 1 lung carcinoma, 1 aspergilloma, 5 bronchiectasis, 1 lung abscess, 1 pneumonia, 1 DPLD and 1 carcinoma of oesophagus.

\section{CONCLUSION}

Bronchoscopy is a useful tool in early diagnosis of smear negative PTB in tertiary care centres like teaching hospitals, where there is availability of resources and skill. It will help in preventing under-diagnosis as well as over-diagnosis of PTB. Even though it is an invasive procedure, its calculated usage in selective patients with diagnostic dilemma will help in diagnosis as well as in treatment. Combined usage of post bronchoscopy AFB along with bronchial washings culture, Gene Xpert may provide better diagnostic yield.

Sensitivity for bronchial aspirates for AFB is $85 \%$ and specificity is $100 \%$. The microscopic examination of bronchial aspirates obtained by FOB is helpful to come to a diagnosis efficiently in smear negative suspected pulmonary TB patients.

\section{REFERENCES}

[1] WHO. Global tuberculosis report, 2014.

[2] WHO unite to end TB, on the road to end TB, 2016. 
[3] Kulpati DD, Heera HS. Diagnosis of smear negative pulmonary tuberculosis by flexible fibreoptic bronchoscopy. Indian J Tuberc 1986;33:179-82.

[4] Balakrishna J, Shahapur PR, Chakradhar P, et al. Role of fiberoptic bronchoscope in sputum smear negative, radiological suspect cases of pulmonary tuberculosis. J Pharm Sci \& Res 2015;7(4):231-3.

[5] Quaiser S, Agarwal A, Khan R, et al. Fiberoptic bronchoscopy, as a valuable diagnostic option in sputum negative pulmonary tuberculosis: a prospective study. International Journal of Applied and Basic Medical Research 2012;2(2):123-7.

[6] Shin JA, Chang YS, Kim TH, et al. Fiberoptic bronchoscopy for the rapid diagnosis of smearnegative pulmonary tuberculosis. BMC Infectious Diseases 2012;12:141.

[7] Khara NV, Kshatriya RM, Vala DH, et al. Diagnostic yield of fiberoptic bronchoscopy (fob) in three common lung conditions at a rural teaching hospital. National Journal of Medical Research 2013;3(4):392-5.
[8] Bachh AA, Gupta R, Haq I, et al. Diagnosing sputum/smear-negative pulmonary tuberculosis: does fibre-optic bronchoscopy play a significant role? Lung India 2010;27(2):58-62.

[9] Mohan A, Sharma SK. Fibreoptic bronchoscopy in the diagnosis of sputum smear-negative pulmonary tuberculosis: current status. Indian J Chest Dis Allied Sci 2008;50(1):67-78.

[10] Thomas A, Gopi PG, Santha T, et al. Predictors of relapse among pulmonary tuberculosis patients treated in DOTS program in South India. Int J Tuberc Lung Dis 2005;9(5):556-61.

[11] Kumar R, Singh M, Gupta N, et al. Bronchoscopy in diagnosis of smear negative tuberculosis. Pneumonol Alergol Pol 2014;82(5):410-4.

[12] Kalawat U, Sharma KK, Reddy PN, et al. Study of bronchoalveolar lavage in clinically and radiologically suspected cases of pulmonary tuberculosis. Lung India 2010;27(3):122-4. 